\title{
Inovação nas empresas de base tecnológica de pequeno porte: um estudo bibliométrico na base Scopus
}

Esse artigo tem como objetivo analisar as produções científicas sobre os indicadores de inovação que caracterizam as Empresas de Base Tecnológica de Pequeno Porte dentro do cenário atual de mercado mundial. Foram apresentadas discussões conceituais sobre empresas de base tecnológica e inovação, utilizando-se para justificar as análises encontradas neste estudo. Dentre os aspectos metodológicos, a pesquisa é exploratória e descritiva, com tratamento qualitativo e quantitativo. Para efeito da pesquisa, a busca das informações foi realizada através da Bibliometria, na base Scopus. Na busca, foram utilizadas as palavras-chave: 'indicadores+inovação+pequenas empresas' e, posteriormente, foi ampliada a pesquisa com as palavras-chave sinônimos e em inglês, para perceber as alteraçõe em termos numéricos e de análises dentro dos critérios de validação de uma string. Os resultados obtidos foram que, apesar de haver discussões sobre conceitos de empresa de base tecnológica e inovação, ainda poucas pesquisas científicas que tratem deste tema. Os estudos resultantes desta pesquisa totalizaram em apenas, 20 artigos, o que motivou a desenvolver um aprofundamento em relação aos indicadores de inovação traçados para as empresas de base tecnológica de pequeno porte. No Brasil, apenas 1 (um) estudo foi realizado pela Universidade Estadual de Maringá em Parceria com a Universidade Positivo, no Paraná.

\section{Innovation in small technological base companies: a bibliometric study based on Scopus}

\begin{abstract}
This article aims to analyze the scientific productions on the innovation indicators that characterize Small Technology-based Companies in the current world market scenario. Conceptual discussions about technology-based companies and innovation were used to justify the analyzes found in this study. Among the methodological aspects, the research is exploratory and descriptive, with qualitative and quantitative treatment. For the purpose of the research, the search for information was carried out through Bibliometrics, on Scopus. In the search, the keywords were used: 'indicators + innovation + small companies' and, later, the search was expanded with the keywords synonyms and in English, to understand the changes in the numerical and analysis terms within the criteria of validation of a string. The results obtained were that, although there are discussions about concepts of technology-based companies and innovation, there is still little scientific research dealing with this topic. The studies resulting from this research totaled only 20 articles, which motivated the deepening of innovation indicators aimed at small technology-based companies. In Brazil, only 1 (one) study was carried out by Universidade Estadual de Maringá in partnership with Universidade Positivo do Paraná.
\end{abstract}

Keywords: Technology-based companies; Innovation; Indicators; Small industries.

\section{Topic: Inovação Tecnológica}

Reviewed anonymously in the process of blind peer.
Received: $17 / 04 / 2021$

Approved: 10/06/2021
Silvia Manoela Santos de Jesus

Universidade Federal de Sergipe, Brasil

http://lattes.cnpq.br/5781099018176442

http://orcid.org/0000-0001-5723-6872

profasilviamanoela@hotmail.com

Antônio Martins de Oliveira Junior (iD)

Universidade Federal de Sergipe, Brasil

http://lattes.cnpq.br/6812943821298890

http://orcid.org/0000-0002-8635-7048

amartins.junior@gmail.com
Referencing this:

JESUS, S. M. S.; OLIVEIRA JÚNIOR, A. M.. Inovação nas empresas de base tecnológica de pequeno porte: um estudo bibliométrico na base Scopus. Revista Brasileira de Administração Científica, v.12, n.2, p.290-299, 2021. DOI: http://doi.org/10.6008/CBPC2179684X.2021.002.0023 


\section{INTRODUÇÃO}

Dada à velocidade em que as tendências de mercado se alteram e os ciclos de vida dos produtos diminuem, torna-se imprescindível para as empresas se envolverem em atividades inovadoras, visando manterem-se competitivas e alcançarem diferenciais perante o mercado (VARGAS et al., 2016).

Em particular, as empresas de base tecnológica (EBT), normalmente possuem como característica a geração de um alto volume de inovações e a absorção de muitos conhecimentos voltados à tecnologia (SPERAFICO et al., 2016). As empresas de base tecnológica consistem em organizações que têm no conhecimento um componente estratégico para a sua competitividade, realizando importantes esforços tecnológicos, por concentrarem significativa parte de sua dedicação no desenvolvimento e fabricação de produtos que integram sistematicamente novas tecnologias (WILLERDING et al., 2016).

O processo de criação ou lançamento de uma empresa de base tecnológica é complexo por causa de sua conexão entre a pesquisa básica, os setores ou nichos de mercado para os quais são direcionados, uma vez que não atingiram sua maturidade plena e a aplicação concreta de tecnologia (VARGAS et al., 2016).

Os resultados conquistados pelas empresas de base tecnológica, através da inovação, consistem basicamente, na criação e o aproveitamento de novas ideias que têm se mostrado como as maneiras mais eficazes das organizações se diferenciarem umas das outras, já que através desta prática é possível perceber as alterações inerentes ao mercado e transformá-las em ações que gerem melhores desempenhos (SILVA et al., 2013).

Devido a essas características, as EBT se tornam, na percepção dos autores supracitados, um campo promissor de pesquisa, no sentido de compreender como os recursos, conhecimentos e tecnologias aplicadas podem alavancar os resultados delas (GENUÍNO et al., 2016). Porém, em meio a discussões e conceitos para definir empresas de base tecnológica (EBT), o problema de pesquisa que surge para ser estudado neste artigo é: como estão os estudos voltados para indicadores de inovação de empresas de base tecnológica de pequeno porte, no mundo?.

Sendo as empresas de base tecnológica as propulsoras para o processo de transferência de tecnologia, este estudo torna-se relevante na medida em que oportuniza a percepção de lacunas que poderiam ser preenchidas no sentido de otimizar o processo de pesquisa e desenvolvimento, que trazem como consequência um direcionamento mais apropriado quanto à identificação de inovação, seus aspectos característicos, seus enfoques principais e tendências. Assim, propõe-se neste artigo, analisar as produções científicas sobre os indicadores de inovação que caracterizam as Empresas de Base Tecnológica de Pequeno Porte dentro do cenário atual de mercado mundial.

\section{METODOLOGIA}

O presente artigo foi realizado a partir de uma pesquisa descritiva e bibliográfica, para reunir as informações e dados que servirão de base para a construção da investigação proposta a partir do tema, ocorrendo análise de registros em documentos de pesquisas realizadas anteriormente (GIL, 2008). O caráter 
da pesquisa é eminentemente exploratório, pois consiste na realização de um estudo para a familiarização do pesquisador com o objeto que está sendo investigado. Já a natureza da pesquisa é do tipo aplicada, pois investigou-se um problema que se refere à aplicação de um conhecimento científico pré-existente (SEVERINO, 2008; MARCONI et al., 2010).

Em relação aos procedimentos adotados, primeiro buscou-se identificar, através do levantamento bibliográfico, quais são os conceitos que dão embasamento teórico ao conceito de empresa de base tecnológica, bem como a identificação de artigos que tratam diretamente desta identificação e que direcionam aos indicadores de inovação. Em um segundo momento, foram analisadas as respectivas produções científicas, dentro da base de dados Scopus.

A pesquisa pode ser caracterizada como exploratória e descritiva com uma abordagem qualitativa e quantitativa (DALFOVO et al., 2008). Para cumprir o objetivo deste estudo, foi realizada uma busca de informações utilizando-se da base de dados Scopus. O presente estudo optou por uma metodologia qualitativa, pois o intuito foi analisar as produções científicas acerca dos indicadores de inovação para as empresas de base tecnológica de pequeno porte (EBTPP).

Para tanto, foi realizada uma análise bibliométrica, que são os estudos que procuram quantificar os produtos da atividade científica (livros, artigos e revistas) para fins de gestão de bibliotecas e bases de dados (SANTOS et al., 2009). Além disso, significa a aplicação de métodos matemáticos e estatísticos a livros e outros meios de comunicação escrita (ALVARADO, 1984). No Brasil, os estudos bibliométricos proliferaram na década de 1970, principalmente com os estudos realizados no Instituto Brasileiro de Bibliografia e Documentação - IBBD, hoje Instituto Brasileiro de Informação Científica e Tecnológica, IBICT. Os estudos bibliométricos realizados nesse período incidiram sobre a literatura científica de vários campos científicos (ARAÚJO, 2006).

Neste estudo, foi inicialmente, levantada a pesquisa com as palavras-chave, em linguagem inglês e português, para encontrar possíveis palavras sinônimas ao estudo, verificar quadro 1.

Quadro 1: Palavras-chave para encontrar string.

\begin{tabular}{|l|l|}
\hline Português & Inglês \\
\hline Indicadores & Indicators \\
\hline Inovação & Innovation \\
\hline Modelo & Model \\
\hline Gestão & Management \\
\hline Tecnologia & Technology \\
\hline Indústria & Industry \\
\hline
\end{tabular}

Tais palavras-chave embasaram a busca para a análise da pesquisa, pois possuem a relevância para atingir a string adequada. A tradução para o inglês se torna necessária, inclusive com a utilização de operadores boleanos, para que consiga restringir, dentro da base Scopus, todas as combinações possíveis. A Scopus foi escolhida pelo seu grau de confiabilidade e por abranger outras áreas, garantindo uma visão ampla de interdisciplinaridade.

Ao final da pesquisa com palavras-chave combinadas, chegou-se a seguinte string: "indicators AND innovation AND model AND management AND technology AND small AND industry". 
Figura 1 apresenta a sequência lógica da busca: as primeiras buscas foram usadas duas palavras-chave (em azul); a segunda busca, também foram utilizadas mais duas palavras-chave (em lilás); já a terceira busca, foram incluídas as quatro palavras-chave que resultaram na string.

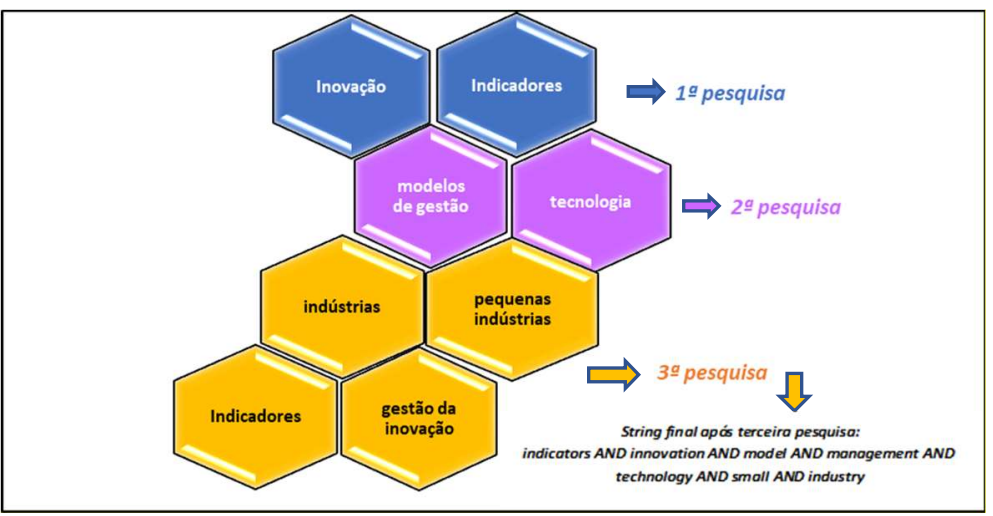

Figura 1: String utilizada para a busca na base da Scopus.

Todos os dados encontrados na busca, foram tabulados em planilhas do Microsoft Excel 2010, para posterior construção de gráficos e análises associadas.

\section{Indicadores de Inovação: conceitos e contribuições para as Empresas de Base Tecnológica de Pequeno Porte (EBTPP)}

Nas duas primeiras décadas do século XXI, não existe termo mais valorizado no mundo dos negócios do que a palavra inovação. Há um reconhecimento crescente da importância da inovação para manutenção e expansão de negócios, e que o investimento em inovação traz retornos efetivos à economia e ao bem-estar da população. Há abordagens que defendem que a competência de inovação é difícil de desenvolver e precisa ser alimentada e protegida constantemente em um ambiente de exploração (TIDD et al., 2015). Nessa perspectiva, com vistas a habilitar competências inovativas de organizações, a metodologia Auditoria de Inovação possibilita avaliar o desempenho empresarial e identificar os pontos que precisam de melhoria em uma empresa (TIDD et al., 2015).

Dentro da promoção de ativos inovativos no âmbito empresarial, alguns estudos mostram que não há relação entre o porte da empresa e sua capacidade efetiva de inovar (ROMÃO, 2017). Porém, Gomes et al. (2018) destaca que sem conhecimento e somado à falta de ferramentas de gestão de inovação impedem que pequenas e médias empresas (PME) possam inovar de forma eficaz.

As empresas de base tecnológica - EBT - constituem para o desenvolvimento do país, pois nelas nascem novas tecnologias, produtos e/ou serviços intensivos em conhecimentos, inovações e podem ser consideradas como propulsoras da pesquisa e desenvolvimento, chamando a atenção da academia para investigações científicas de várias áreas do conhecimento (VARGAS et al., 2016; OSUNA et al., 2016). Sua importância, como espaço de atuação profissional, é reconhecida por cientistas há muito tempo e, talvez por isso, os estudiosos foram os primeiros que se debruçaram sobre as especificidades de tais empresas (WILLERDING et al., 2016), onde a capacidade de inovar desempenha papel essencial (TELLO et al., 2016).

Para Drucker (2016), a organização moderna necessita ser orientada para a inovação, para o 
abandono sistemático daquilo que é rotineiro, conhecido e confortável, seja produto, processo, serviço, conjunto de aptidões, recursos humanos ou a própria organização. Para o autor, a nova função da organização é, então, aplicar novos conhecimentos ao trabalho, atividade que, naturalmente, se faz necessária, entretanto, precisa ser renovada rapidamente. Marcuzzo et al. (2015) reconhecem que as EBT dominam esses novos conhecimentos, diretrizes e objetivos fundamentais que favorecem o surgimento de novos paradigmas a serem "quebrados", como a gestão do conhecimento nas organizações e, dessa forma, a aprendizagem organizacional constituir-se-á de uma forte arma para a competitividade.

Como já mencionado nesta tese, as EBT precisam de uma gestão solidificada na inovação e gestores competentes para que seus projetos tenham fluidez, rapidez e flexibilidade para identificar oportunidades e antecipar mudanças, lembrando que estas são consideradas organizações cujas atividades empresariais na inovação contam com pessoal investigador e técnico de alta qualificação, mas seu sucesso dependerá de outros fatores (ROSA, 2019).

O termo inovação traz em sua base os preceitos de Chesbrough e seus seguidores. Demarchi et al. (2016), afirmam que inovar significa ter uma ideia nova ou, aplicar a ideia de outros de uma forma original e com eficácia. Porém, não é só isso. Trata-se de uma nova ideia aliada à implementação, ou a exploração com sucesso de novas ideias. Assim, Demarchi et al. (2016) dizem que o termo inovação é empregado em pelo menos três diferentes contextos: sinônimo de invenção: refere-se a um processo criativo em que dois ou mais conceitos são combinados de uma forma nova para produzir uma configuração não conhecida; uma mudança: algo, processo e ou procedimento novo para a organização; e na visão popular algo prático ou artefato material: algo inventado ou visto como novo independente de sua adoção.

De acordo com a Lei da Inovação 10.973 de 2004, inovação, então, é a concepção de novo produto ou processo de fabricação, bem como a agregação de novas funcionalidades ou características ao produto ou processo que implique em melhorias incrementais e efetivo ganho de qualidade ou produtividade, resultando maior competitividade no mercado.

Se a introdução de novos produtos e processos baseados em tecnologia no mercado é uma das principais características que definem as pequenas empresas de base tecnológica, é necessário compreender não somente quais inovações elas introduzem no mercado, mas também de que forma essas empresas executam as atividades que as levam à criação e comercialização de inovações tecnológicas.

As empresas que buscam inovar, independentemente do porte ou do nível de formalidade do processo, devem seguir estas quatro grandes etapas para introduzir inovações no mercado. Inicialmente, devem buscar as oportunidades disponíveis, selecionar algumas destas oportunidades, empreender esforços para implementar - onde incluímos as atividades de P\&D - e por fim levar o novo produto ou serviço para o mercado. Em pequenas empresas, é possível que esta atividade não seja estruturada formalmente, pois as pequenas empresas podem apresentar certas lacunas de conhecimento gerencial ou não dispor de pessoas com formação e experiência adequadas para executar todas estas atividades de maneira formal (VALLONE, 2017).

De acordo com levantamento de pesquisas, pode-se assumir que os setores de maior intensidade 
tecnológica (produtos elétricos, maquinário, produtos químicos e equipamentos de transporte) contenham uma fração expressiva de EBTPP, as quais fazem relativamente maiores dispêndios em P\&D (RAYMOND et al., 2010). Para gerar e comercializar suas inovações, as EBTPP necessitam de recursos, habilidades e rotinas que permitam a elas transformar conhecimentos tecnológicos em novos produtos e serviços. Neste processo de inovação, estas empresas apresentam tanto vantagens quanto desvantagens em relação à inovação em comparação com as grandes empresas.

Se por um lado as pequenas empresas são mais ágeis em responder aos sinais do mercado, por outro elas podem ter restrições para introduzir estes novos produtos, processos e tecnologia no mercado devido à limitação de recursos internos. Especificamente em relação a EBTPP, espera-se que elas sejam mais intensivas em P\&D do que as demais categorias de pequenas empresas, contem com mão de obra especializada, e recorram com frequência aos inputs de conhecimentos científicos e de clientes, utilizandose de cooperação em suas atividades de inovação. As atividades de inovação nestas empresas podem ser também influenciadas pelo contexto nacional. Em países em desenvolvimento como o Brasil, é possível que haja deficiências do Sistema Nacional de Inovação para o suporte às EBTPP, o que pode agravar as dificuldades típicas destas empresas (VALLONE, 2017).

A definição dos indicadores se torna importante para o sucesso de uma empresa, já que eles podem ser usados como ferramentas para se traçar estratégicas em níveis, departamentos e, até mesmo, localidades de uma mesma organização. Os indicadores são sinais vitais da organização que qualificam e quantificam o modo como as atividades inovadoras ou saídas de um processo atingem suas metas. São características de um bom indicador: a) bem definido, simples, claro, compreensível, sem interpretação dúbia; b) pertinente, significativo e sensível a variações; c) presente e real; d) disponível e fácil de ser utilizado; e) fiel ao objetivo pelo qual foi definido, confiável e homogêneo às variações de tempo e espaço passível de ser agregado e comparado (CARDOSO et al., 2018).

\section{RESULTADOS E DISCUSSÃO}

A inovação em uma economia globalizada vem ganhando destaque pela contribuição na competitividade, crescimento e rentabilidade das empresas. Inovar envolve estratégia, processos, cultura e clima organizacional, desde inovações incrementais em produtos e serviços até inovações que modifiquem o modelo de gestão ou de negócios da empresa.

Inovar é unir o desejo do consumidor com o que é possível pela tecnologia. Para tanto, é necessário acessar os melhores talentos e recursos disponíveis, em qualquer lugar do mundo. Realizar a gestão dos processos de inovação é essencial para gerar resultados. Neste estudo, conforme sinalizado na metodologia, a Bibliometria auxiliou a percepção de pesquisas destinadas à inovação em EBTPP. À medida em que as palavras-chave foram utilizadas em sua combinação, percebe-se uma regressão na quantidade de publicações científicas, relacionadas à temática, com o percentual de $0,2 \%$ ao se comparar com a primeira combinação entre indicadores e inovação. 0 quadro 2 destaca esta informação. 
Quadro 2: Mapeamento das publicações científicas de indicadores de inovação nas empresas de base tecnológica de pequeno porte - EBTPP.

\begin{tabular}{|l|l|l|}
\hline Combinação & Strings & Resultados \\
\hline 1 & Indicators and Innovation & 9.976 \\
\hline 2 & Indicators and Innovation and model & 2.932 \\
\hline 3 & Indicators and Innovation and model and Management & 1.175 \\
\hline 4 & Indicators and Innovation and model and Management and Technology & 349 \\
\hline 5 & Indicators and Innovation and model and Management and Technology and Industry & 128 \\
\hline 6 & Indicators and Innovation and model and Management and Technology and Small Industry & 20 \\
\hline
\end{tabular}

Conforme informação do quadro 2, houve uma quantidade significativa (iniciando com 9.976 publicações científicas), porém, chegou-se a apenas 20 artigos, relacionados aos indicadores de inovação para as empresas de base tecnológica de pequeno porte. Destes 20 artigos encontrados, foi investigada a quantidade de publicações por ano, estimando-se os últimos 10 anos. 0 resultado da investigação está apresentado na Figura 2.

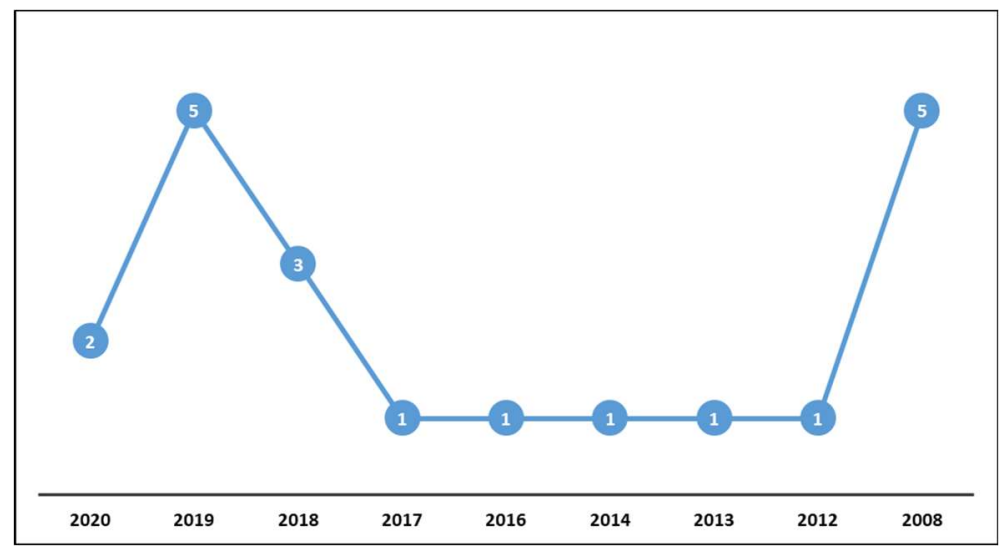

Figura 2: Publicações ao longo dos anos 2008 a 2020.

A figura 2 mostra que os anos de 2008 e 2019 apontam uma participação mais relevante diante dos demais, porém, uma quantidade pequena, o que motivou a pesquisa em tratar desta temática. Nestes anos, a inovação despontou de forma mais significativa. O início da pandemia em 2019 estimulou as empresas, principalmente as pequenas, a se reinventarem em um cenário atípico. Tão importante quanto inovar é saber como inovar, dessa forma, pesquisar, analisar e gerar conhecimento para tomada de decisão tem se constituído uma das principais atividades para as organizações que querem se manter competitivas no mercado. O recurso "conhecimento" e os desafios impostos pela abertura econômica tornam a gestão desse conhecimento ainda mais fundamento para as empresas brasileiras (RAYMOND et al., 2010).

Destas publicações, houve a representação, na sua maioria, em países europeus, porém, também há estudos latinos, e, inclusive, no Brasil, da Universidade de Maringá. Conforme já sinalizado, com a combinação da string, a quantidade relativa pequena, tem 1 publicação em cada Instituição, de acordo com a Figura 3. Vale ressaltar que foram sinalizadas aqui apenas 10, das 20 afiliações, escolhidas pelos autores pela relevância da produção para este estudo. Destaque para o artigo brasileiro, construído pela parceria entre pesquisadores da Universidade Estadual de Maringá e Universidade Positivo, que tratou de investigar os modelos de negócios e a influência das pressões ambientais no resultado inovador de indústrias. A resposta foi representada por modelo, focado na eficiência ou focado em inovação. 


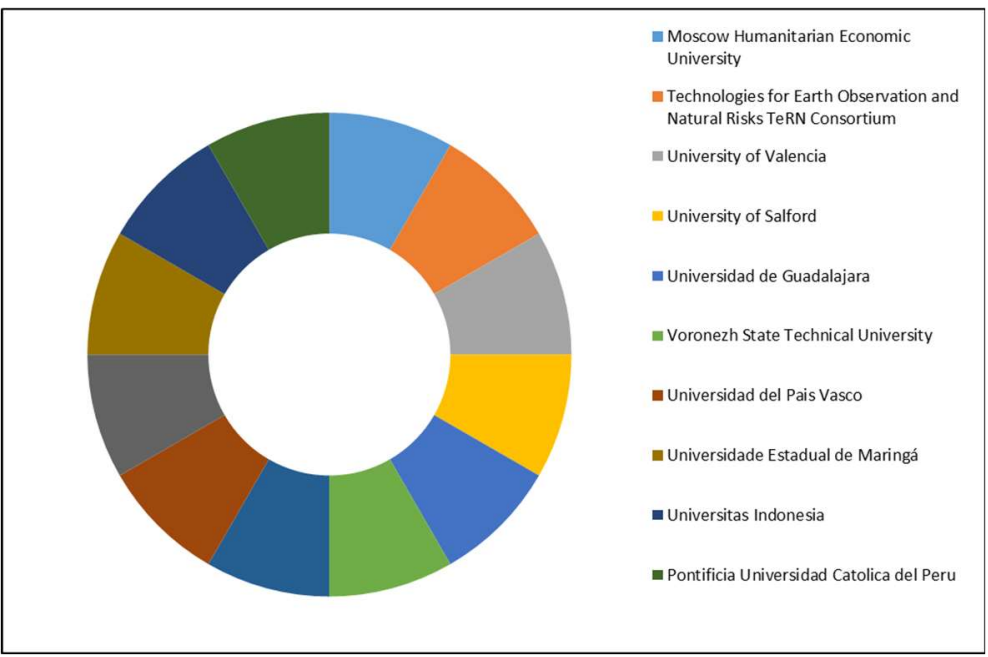

Figura 3: Afiliação das publicações.

Os artigos mais citados foram os da Universidade de Guadalajara e University of Valencia, com 13 e 42 citações, respectivamente. Ambos trataram em seus estudos sobre os indicadores de inovação nas indústrias, garantindo a competitividade. Assim, os indicadores de inovação são parâmetros selecionados, considerados individualmente ou combinados entre si, utilizados para avaliar e analisar determinadas condições dos dados obtidos nestes sistemas (BARBOSA et al., 2013). Dentre as palavras-chave mais utilizadas nestes estudos, as mais relevantes estão na figura 4.

Estas palavras-chave coadunam-se com as palavras utilizadas para a definição da string deste estudo, conforme já explicitado na figura 1. Embora as discussões sobre inovação sempre estejam associadas à criatividade, eficiência, lucratividade, estes indicadores são inerentes às grandes empresas. Nas pequenas indústrias, que representam uma importante movimentação no mercado, não se atentam para a inovação em sua essência. Para elas, basta ter um diferencial que agregue valor ao cliente, mas, seus processos, em sua maioria, não pratica a inovação. Por isso que quando foi construída a string, as palavras-chave 'comuns' estavam presentes.

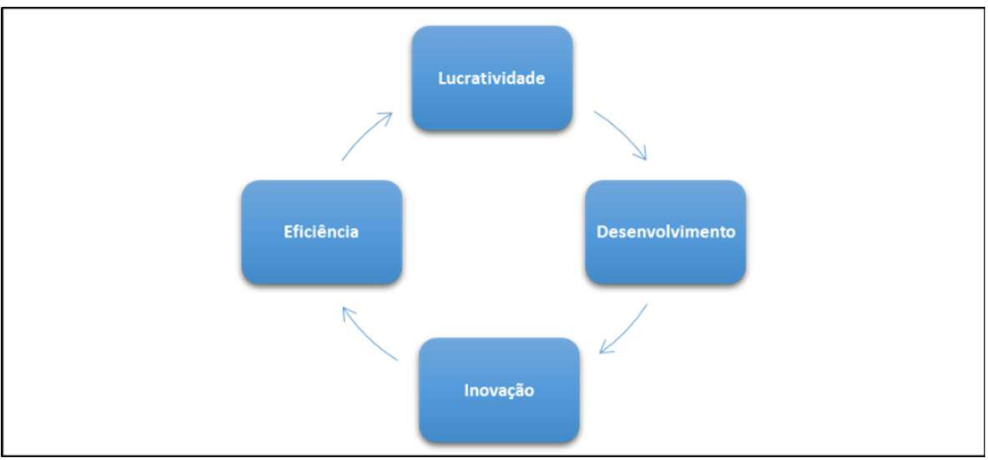

Figura 4: Palavras-chave citadas nas produções pesquisadas.

Alguns autores, sugerem que o processo de inovação pode ser definido como um conjunto de estágios que parte da invenção para posterior desenvolvimento, produção, introdução ao mercado, onde ocorre a difusão inovativa propriamente dita, e é plausível que tal difusão seja entendida como o processo de disseminação no sistema social da nova tecnologia desenvolvida (DUARTE-SILVA, 2017).

A partir disso, seguem informações relacionadas aos países, autores, coautores e número de 
publicações (Quadro 3). Este quadro apresenta uma relação de confirmar que, no geral, os 20 artigos representaram uma variedade de nacionalidades e estudos na América Latina e Europa, mas, em quantidade, apenas 1 artigos por autoria/coautoria.

Quadro 3: Levantamento de participações em publicações na pesquisa.

\begin{tabular}{|l|l|l|}
\hline Autor e Coautor & País & Publicação \\
\hline Annunziato, M. & Itália & 1 \\
\hline Braccio, G. & Itália & 1 \\
\hline Cabanelas, P. & Chile & 1 \\
\hline Cornacchia, C. & Peru & 1 \\
\hline Hernández, I. M. V. & Peru & 1 \\
\hline Di Renzo, G. C. & Itália & 1 \\
\hline Silva, F. M. & Brasil & 1 \\
\hline Roberto Dos Reis, D. & Brasil & 1 \\
\hline Salvia, M. & Rússia & 1 \\
\hline Sánchez-Gutiérrez, J. & Guadalajara & 1 \\
\hline
\end{tabular}

Assim, vale ressaltar que, onde se repetem os países, significa que o artigo foi produzido em autoria e coautoria, resultando em 1 artigo. Por exemplo, os autores Meller da Silva e Roberto dos Reis, publicaram o mesmo artigo brasileiro (1), sendo um autor e outro coautor desta produção científica.

\section{CONCLUSÕES}

Estudos sobre indicadores são importantes, pois estabelecem uma correlação entre as diretrizes estratégicas e práticas da realidade, de modo que se possa assegurar que essas mesmas diretrizes estejam incorporadas nos processos da organização. Segundo Frederiksen et al. (2005), também são úteis para descrever e avaliar situações atuais, planejar atuações futuras, ou comparar duas situações em períodos diferentes, auxiliando na compreensão de uma ideia de origem complexa.

Neste estudo, foi feito o levantamento de pesquisas científicas sobre os indicadores de inovação para as pequenas indústrias, por meio da análise bibliométrica na base Scopus. Para a análise através da string final, foram encontrados 20 artigos, porém, que se alinhavam com a objetivo traçado neste estudo. Inicialmente, foi dado como surpresa este resultado, visto que a inovação vem se destacando em todos os setores e segmentos na economia mundial, mas, dentro do cenário de pequenas indústrias, não mantem a mesma linha de estudos.

As pesquisas foram relativamente pequenas no sentido de número. Cada afiliação produziu 1 (um) artigo. No Brasil, houve a representação da parceria entre a Universidade Estadual de Maringá (UEM) e a Universidade Positivo (UP), no Paraná. Houve também estudos latinos, como no Peru, Chile e, outros na região da Itália (Valencia, por exemplo).

Tal fato trouxe aos autores a motivação de aperfeiçoar as investigações para compor um modelo de indicadores apropriados para as pequenas indústrias, considerando suas especificidades de porte, mas não sendo limitantes a algum segmento ou setor. As pesquisas foram claras: a inovação existe para as empresas de médio e grande portes, sendo, inclusive, ponto relevante para traçar estratégias de manutenção e crescimento no mercado competitivo.

Portanto, as empresas de base tecnológica precisam se modernizar, mesmo que, paulatinamente, 
pela constante difusão de novas tecnologias, do trabalho de incubadoras de empresas e de outras formas que permitam a transformação tecnológica a médio e longo prazos.

\section{REFERÊNCIAS}

ALVARADO, R. U.. A Bibliometria no Brasil. Ciência da Informação, v.13, n.2, p.11, 1984.

BARBOSA, E. B.; PIMENTA, H. F.; CASTRO, A. P.. Indicadores de sustentabilidade e sua dimensão ambiental: ESI, EPI, LPI, Pegada Ecológica, BIP 2020. Revista Desarrollo Local Sostenible (DELOS), v.16, n.18, p.1-9, 2013.

BRASIL. Lei n. 10.973, de 02 de dezembro de 2004. Dispõe sobre incentivos à inovação e à pesquisa científica e tecnológica no ambiente produtivo e dá outras providências. Brasília: DOU, 2004.

CARDOSO, A. F.; SOUZA, V.; HOELTGEBAUM, M.. Análise Comparativa dos Indicadores de Desempenho em Pequenas Empresas. In: SIMPÓSIO DE EXCELÊNCIA EM GESTÃO E TECNOLOGIA, 3. Anais. 2018. p.1-12.

DALFOVO, M. S.; LANA, R. A.; SILVEIRA, A.. Métodos quantitativos e qualitativos: um resgate teórico. Revista Interdisciplinar Científica Aplicada, v.2, n.3, p.1-13, 2008.

DEMARCHI, A. P. P.; FORNASIER, C. B. R.; MARTINS, R. F. F. Design Thinking e seus códigos visuais na gestão de design para inovação: Inovação incremental pelo modelo GEIDA. DAPesquisa, v.11, n.16, p.191-211, 2016.

DRUCKER, P.. Inovação e espírito empreendedor: práticas e princípios. São Paulo: Cengage Learning, 2016.

DUARTE-SILVA, M.. Inovação em pequenas e médias empresas: uma análise comparativa entre Brasil e países europeus. 2017.

FREDERIKSEN, H.; MATHIASSEN, L.. Information-centric assessment of software metrics practices. IEEE Transactions on Engineering Management, v.52, n.3, p.350-362, 2005.

GEnuínO, S. L. V. P.; MACHADO, A. G. C.. Pesquisa \& desenvolvimento com inovação aberta: o caso EMEPA. Gestão \& Aprendizagem, v.4, n.2, p.56-75, 2016.

GIL, A.. Como elaborar projetos de pesquisa. São Paulo: Atlas, 2008.

GOMES, E.; NIRAZAWA, A. N.. Auditoria tecnológica de inovação: contribuições para pequenas e médias empresas. Cadernos de Gestão e Empreendedorismo, Ribeirão Preto, v.6, n.2, 2018.

MARCONI, M.; LAKATOS, E.. Fundamentos da metodologia científica. São Paulo: Atlas, 2010.

MARCUZZO, R.; SILUK, J.. Proposta para criação de diagnóstico de evidenciação dos fatores intangíveis de desempenho em empresas de base tecnológica. In: SAEPRO, 10. Anais. Viçosa, 2015.

OSUNA, M. A. A.; PÉREZ, C. D. C. D.. La empresa de base tecnológica y su contribución a la economía mexicana en el periodo 2004-2009. Contaduría y Administración, v.61, n.1 p.106-126, 2016

RAYMOND, L.; ST-PIERRE, J. R. D.. As a determinant of innovation in manufacturing SMEs: An attempt at empirical clarification. Technovation, v.30, n.1, p.48-56, 2010.

ROMÃO, H. R. S.. Auditoria da inovação: estudo de caso sobre o programa agente de inovação - ALI. Dissertação (Mestrado em Administração de Empresas) - Centro Universitário FEI, São Paulo, 2017.

ROSA, A. C. M.. Modelo de referência para inovação aberta em empresas de base tecnológica. 2019.

SANTOS, R. N. M.; KOBASHI, N. Y.. Bibliometria, cientometria, infometria: conceitos e aplicações. 2009.

SILVA, G; DACORSO, A. L. R.. Inovação aberta como uma vantagem competitiva para a micro e pequena empresa. RAI Revista de Administração e Inovação, v.10, n.3, p.251-269, 2013.

SPERAFICO, J. H.; ENGELMAN, R.; GONÇALVES, M. A.. Capital intelectual organizacional e inovação em Micro e Pequenas Empresas de base tecnológica. Revista Raunp, v.9, n.1, p.5161, 2016. DOI: http://dx.doi.org/10.21714/raunp.v9i1.1596

TELLO, E. A.; VELASCO, J. M. A. P.. Inteligencia de negocios: estrategia para el desarrollo de competitividad en empresas de base tecnológica. Contaduría y Administración, v.61, n.1, p.127-158, 2016.

TIDD, J.; BESSANT, J.. Gestão da inovação. 5 ed. Porto Alegre: Bookman, 2015.

VALLONE, A. F.. Cooperação em inovação e desempenho de pequenas empresas de base tecnológica: uma análise exploratória a partir da PINTEC. 2017.

VARGAS, M. A. A.; RIOS, B. L. F.; ESQUER, J. E. I.; MARISCAL, J.; CORRAL, L. E. V.. Impacto del aprendizaje basado en proyectos implementado en una empresa escolar de Base Tecnológica dedicada al desarrollo de Software. ReCIBE, v.4, n.4, 2016

WILLERDING, I. A. V.; KRAUSE, M. G.; LAPOLLI, É. M.. Gestão de pessoas e gestão do conhecimento à luz da estética organizacional. Perspectivas em Gestão \& Conhecimento, v.6, n.1, p.141-154, 2016

A CBPC - Companhia Brasileira de Produção Científica (CNPJ: 11.221.422/0001-03) detém os direitos materiais desta publicação. Os direitos referem-se à publicação do trabalho em qualquer parte do mundo, incluindo os direitos às renovações, expansões e disseminações da contribuição, bem como outros direitos subsidiários. Todos os trabalhos publicados eletronicamente poderão posteriormente ser publicados em coletâneas impressas sob coordenação da Sustenere Publishing, da Companhia Brasileira de Produção Científica e seus parceiros autorizados. Os (as) autores (as) preservam os direitos autorais, mas não têm permissão para a publicação da contribuição em outro meio, impresso ou digital, em português ou em tradução. 